2. Über freie Faktorgruppen und freie Untergruppen gegebener Gruppen, Monatshefte für Mathematik und Physik vol. 47 (1938) pp. 307-313.

A. Malcew

1. On isomorphic matrix representations of infinite groups, Rec. Math. (Mat. Sbornik) vol. 8 (1940) pp. 421-422 (405-421).

UNIVERSITY OF ILLINOIS

\title{
A SUBSTITUTE FOR THE AXIOM OF CHOICE
}

\section{A. D. WALlace}

The following result appeared in the 1914 edition of Hausdorff's Mengenlehre, p. 140:

(A) Any partially ordered system contains a maximal simply ordered subsystem.

This theorem is well known to be equivalent to the axiom of choice (though there does not seem to be a proof of this fact in the literature) and it has been suggested as an alternative for this axiom. The purpose of this note (which is purely methodological) is to propose a simpler but equivalent formulation of (A) as a substitute for the Zermelo axiom. The simplicity lies in the fact that we make no assumptions concerning the relation $R$ which replaces partial order.

Let $Q$ be a set and $R$ an arbitrary binary relation on $Q$. A subset of $Q$ will be termed $R$-simple if for any pair of its elements, $a$ and $b$, we have either $a R b$ or $b R a$. The version of (A) we propose is:

(B) Any R-simple subset of $Q$ is contained in a maximal $R$-simple subset of $Q$.

It is clear that (B) implies (A). Conversely, let $Q_{0}$ be an $R$-simple subset of $Q$. Let $P$ be the partially ordered (by inclusion) system composed of all $R$-simple subsets of $Q$ which contain $Q_{0}$. Then by (A) there is a maximal simply ordered subsystem $P_{0}$ of $P$. The union of all the sets in $P_{0}$ is the desired maximal $R$-simple subset.

The University of Pennsylvania

Received by the editors December 12, 1943. 